\title{
Correction to: Correlation between polio immunization coverage and overall morbidity and mortality for COVID-19: an epidemiological study
}

\author{
Marwa Adel Afify ${ }^{1}$ (D) $\cdot$ Rakan M. Alqahtani ${ }^{2} \cdot$ Mohammed Abdulrahman Mohammed Alzamil $^{3}$. \\ Faten Abdulrahman Khorshid ${ }^{4}$. Sumayyah Mohammad Almarshedy ${ }^{5}$. Sana Ghazi Alattas ${ }^{6} \cdot$ Talal Nabeel Alrawaf $^{7}$. \\ May Bin-Jumah ${ }^{8} \cdot$ Mohamed M. Abdel-Daim $^{9,10} \cdot$ Mohammad Almohideb $^{11}$
}

Published online: 5 June 2021

(C) Springer-Verlag GmbH Germany, part of Springer Nature 2021

\section{Correction to: Environmental Science and Pollution Research} https://doi.org/10.1007/s11356-021-12861-6

The correct affiliation of Rakan M. Alqahtani is presented in this paper.

Publisher's note Springer Nature remains neutral with regard to jurisdictional claims in published maps and institutional affiliations.

The original article has been corrected.

The online version of the original article can be found at https://doi.org/ 10.1007/s11356-021-12861-6

Marwa Adel Afify

marwa.adel.pharmacy2@gmail.com

$\triangle$ Mohammed Abdulrahman Mohammed Alzamil

Mohammed.alzamil@yahoo.com

Rakan M. Alqahtani

arakan@ksu.edu.sa

Faten Abdulrahman Khorshid

Fatenkhorshid@yahoo.com

Sumayyah Mohammad Almarshedy

dr.sumayyahalmarshedy@hotmail.com

Sana Ghazi Alattas

sgalattas@kau.edu.sa

\author{
Talal Nabeel Alrawaf \\ t.n.rawaf@gmail.com \\ May Bin-Jumah \\ may_binjumah@outlook.com \\ Mohamed M. Abdel-Daim \\ abdeldaim.m@vet.suez.edu.eg \\ Mohammad Almohideb \\ Moalm20@gmail.com
}

Extended author information available on the last page of the article 


\section{Affiliations}

Marwa Adel Afify ${ }^{1}$ (D) $\cdot$ Rakan M. Alqahtani $^{2} \cdot$ Mohammed Abdulrahman Mohammed Alzamil $^{3}$.

Faten Abdulrahman Khorshid ${ }^{4}$. Sumayyah Mohammad Almarshedy ${ }^{5}$ Sana Ghazi Alattas ${ }^{6}$ - Talal Nabeel Alrawaf ${ }^{7}$. May Bin-Jumah ${ }^{8}$. Mohamed M. Abdel-Daim ${ }^{9,10}$. Mohammad Almohideb ${ }^{11}$

1 Potion CRO, Integrative Medicine Company, Al Malqa, Riyadh 13524, Saudi Arabia

2 Department of Critical Care Medicine, College of Medicine, King Saud University, Riyadh, Saudi Arabia

3 Prince Sultan Medical Military City (PSMMC), Riyadh, Saudi Arabia

4 Department of Biological Sciences, Faculty of Science, King Abdulaziz University, Jeddah 21589, Saudi Arabia

5 Division of Adult Neurology, Department of Internal Medicine, College of medicine, University of Hail, Hail, Saudi Arabia
6 Biological Sciences Department, King Abdulaziz University, Jeddah, Saudi Arabia

7 Al Yamamah Hospital, Riyadh, Saudi Arabia

8 Biology Department, College of Science, Princess Nourah Bint Abdulrahman University, Riyadh, Saudi Arabia

9 Department of Zoology, Science College, King Saud University, Riyadh 11451, Saudi Arabia

10 Pharmacology Department, Faculty of Veterinary Medicine, Suez Canal University, Ismailia 41522, Egypt

11 College of Medicine, King Saud bin Abdulaziz University for Health Sciences, Riyadh, Saudi Arabia 